\title{
Caracterização do gás biogênico da Baía de Aratu.
}

Gabriela Nunes Turquetti1,2, Priscila Martins Oliveira ${ }^{1,2}$, Marcello Pellicano Falleiros ${ }^{1}$, Marco lanniruberto'.

${ }^{1}$ Instituto de Geociências - Universidade de Brasília, ${ }^{2}$ Phygeo Soluções em Geofísica e Geotecnologia

Copyright 2016, SBGf - Sociedade Brasileira de Geofísica

Este texto foi preparado para a apresentação no VII Simpósio Brasileiro de Geofísica, Ouro Preto, 25 a 27 de outubro de 2016. Seu conteúdo foi revisado pelo Comitê Técnico do VII SimBGt, mas não necessariamente representa a opinião da SBGf ou de seus associados. É proibida a reprodução total ou parcial deste material para propósitos comerciais sem prévia autorização da SBGf.

\section{Resumo}

A presença de matéria orgânica aliada a condições de temperatura e pressão propiciam a formação de gás metano nos sedimentos. O presente trabalho teve como objetivo a identificação e mapeamento da ocorrência de gás biogênico na Baía de Aratu (BA) utilizando-se dos métodos geofísicos aquáticos aplicados a ambientes submersos rasos. As investigações geofísicas foram baseadas a partir dos resultados obtidos com o sonar de varredura lateral e com o perfilador de subfundo. Os resultados permitiram identificar evidências visuais que correlacionam com a exsudação de gases na lâmina d'água e em profundidade, como o mascaramento acústico, típico dessas ocorrências.

\section{Introdução}

O metano é produzido nos oceanos de duas formas principais: biogênicamente ou termogênicamente. Um pré-requisito essencial para a formação de metano nos sedimentos marinhos, seja a partir de processos biogênicos ou termogênicos, é a abundância de no mínimo 0,5\% de matéria orgânica (JUDD, 2004). O metano termogênico é produzido em grandes profundidades nos oceanos, quando a matéria orgânica é decomposta pelo calor da Terra. O metano biogênico é produzido a partir das atividades metabólicas de um grupo de arqueobactérias chamado metanogênicos.

A Baía de Aratu (Figura 1), área de estudo deste trabalho, está situada a nordeste da Baía de Todos os Santos (BTS), possui uma área de aproximadamente $24,5 \mathrm{~km}^{2}$. É caracterizada por ser um ambiente de águas calmas de baixa energia, composta basicamente por sedimentos terrígenos e biogênicos (HATJE; DE ANDRADE, 2009). Segundo BITTENCOURT et al. (1974), a Baía de Aratu possui uma predominância de material fino, na faixa argilo-siltoso com áreas marcadamente argilosas. Há também grandes áreas de material grosso associadas a depósitos de conchas e fragmentos esqueletais que foram considerados de formação local, com pequeno transporte. Uma atualização da distribuição espacial das litofácies da BTS é proposta por (LESSA; DIAS, 2009), em que o autor acrescenta litofácies silte e areia em Aratu, bem como suas subdivisões.

Trabalhos anteriores comprovam a exsudação de gases em Aratu cuja natureza é atribuída a atividade biogênica (CAMPOS, 2010; DIAS, 2003; HATJE; DE ANDRADE, 2009). Os sedimentos finos que se acumulam na metade norte da BTS se acumulam em águas rasas e ambientes costeiros, os quais são caracteristicamente ricos em matéria orgânica. Como os sedimentos e rochas sedimentares que apresentam exsudação de gás são finamente granulados, eles são por consequência impermeáveis, facilitando a acumulação de gás.

Evidências visuais nos perfis sísmicos e nas imagens sonográficas da presença de gases em camadas sedimentares rasas são apresentadas por (JUDD et al., 1997). Nos perfis sísmicos pode-se citar: turbiditos acústicos - reflexões caóticas causadas pelo espalhamento da energia; reflexões intensificadas; perturbação colunar - destruição de padrões verticais de reflexão graças à migração do fluido nos poros; e a evidência mais típica, mascaramento acústico - palidez ou ausência de reflexões nas janelas de liberação de gás. Nas imagens sonográficas, são apresentadas feições que indiretamente podem estar relacionadas a presença de gases. As mais comuns são as plumas de infiltrações - plumas de liberação de gás que aparecem semelhantes a um cardume de peixes ou como alvos escuros isolados na coluna d'água; e feições altamente refletivas - marcadas por corpos isolados de coloração escura, devido ao elevado espalhamento do sinal acústico.

Há registros da utilização dos métodos geofísicos sísmicos e sonográficos em estudos de escapes de gás desde a época de exploração oceânica, durante o período pós Segunda Guerra Mundial (DAVIS, 1992). Desde então sua popularidade só tem aumentado, graças aos benefícios do emprego de metodologias multidisciplinares. Este trabalho objetiva identificar áreas que apresentam liberação de gás na Baía de Aratu embasando-se nos resultados obtidos com a geofísica rasa.

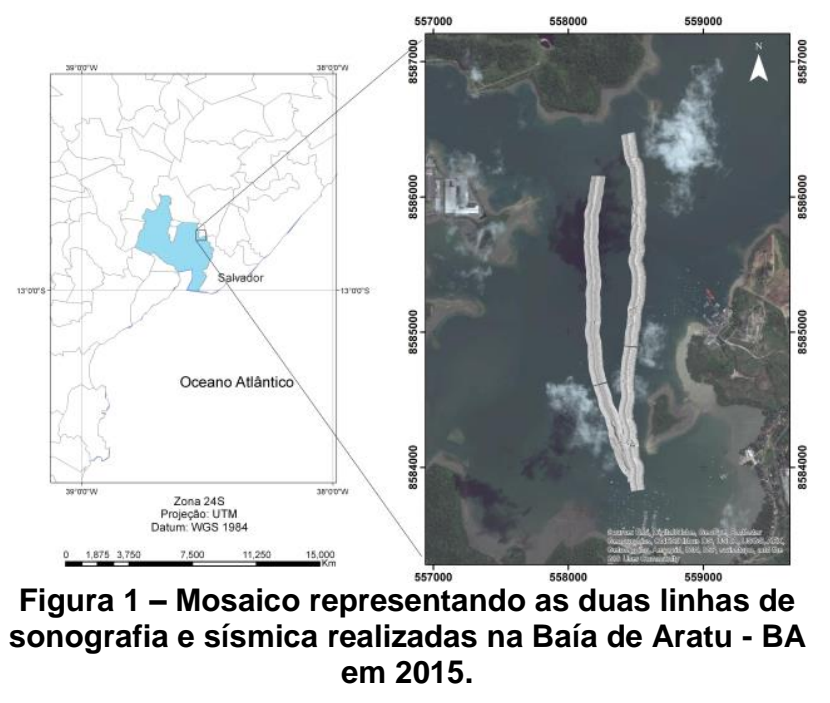




\section{Metodologia}

A fim de identificar e caracterizar o gás biogênico da Baía de Aratu, foram adquiridas linhas de aproximadamente 3 quilômetros cada no sentido NS com o Sonar de Varredura Lateral e o Perfilador de Subfundo (Figura 1).

Observa-se na literatura o emprego conjunto da sonografia e da perfilagem sísmica de alta resolução em ocorrências de exsudação de gás (CAMPOS, 2010; JUDD; HOVLAND, 1992; JUDD, 2004). A propagação e penetração das ondas sísmicas em profundidade na perfilagem sísmica possibilita a caracterização das camadas sedimentares, bem como do embasamento rochoso, graças à visualização em perfil. As imagens sonográficas do fundo podem trazer resultados interessantes sobre as estruturas e feições que possam estar correlacionadas com a exsudação do gás, além de possibilitar uma caracterização textural da distribuição sedimentar superficial.

Para a investigação geofísica sonográfica empregou-se o Side Scan Sonar Edgetech 4100 com sensor TD272 a uma frequência de $400 \mathrm{kHz}$, e para a sísmica de alta resolução o perfilador acústico de subfundo Edgetech $3100 \mathrm{P}$ com uma frequência de 2 a $15 \mathrm{kHz}$, ambos georeferenciados pelo GPS Hemisphere A325. O software utilizado para a aquisição dos dados foi o fornecido pelo fabricante e para navegação utilizou-se o Hypack.

O processamento dos dados foi realizado com o auxílio dos softwares Sonarwiz para os dados do Sonar de Varredura Lateral e GeoSuite para os dados do Perfilador de Subfundo. O processamento dos dados consistiu no realce do sinal, com a aplicação de ganhos e filtros para melhorar a visualização dos dados e conseguinte interpretação.

\section{Resultados e Discussões}

Entender o comportamento acústico do gás é muito importante para uma interpretação geofísica coerente. Em geral a resposta acústica do gás está diretamente relacionada com a quantidade de bolhas e os raios das mesmas que normalmente possuem um comprimento entre 0.1 e $0.55 \mathrm{~cm}$ (JUDD et al., 1997; LEIFER AND MACDONALD, 2003). Mesmo em pequenas concentrações, bolhas de gás existentes no sedimento ou na coluna d'água dissipam a energia acústica (fenômeno conhecido como difusão), podendo aumentar o retroespalhamento, causando uma marcada resposta nos perfis sísmicos rasos (CAMPOS, 2010). Os resultados desse trabalho são apresentados nas Figuras $2 \mathrm{~A}$ e $2 \mathrm{~B}$ referentes aos dados obtidos para o Perfilador de Subfundo e as Figuras $3 \mathrm{~A}, 3 \mathrm{~B}$ e $3 \mathrm{C}$ referentes à resposta do Sonar de Varredura Lateral.

O alcance vertical máximo obtido com o perfilador sísmico foi de $35 \mathrm{~m}$. Os registros sísmicos possibilitou individualizar as regiões que apresentam exsudação de gases graças as evidências propostas por (JUDD et al., 1997). É possível observar o mascaramento acústico completo do refletor subjacente à zona de liberação de gases em janelas ao longo do perfil. Este mascaramento está presente em regiões onde os pacotes sedimentares possuem uma espessura aproximadamente maior ou igual a $5 \mathrm{~m}$ (Figura 2B).

Segundo a classificação apresentada por (GARCIA-GIL; VILAS; GARCIA-GARCIA, 2002) e Iglesias e Garcia-Gil (2007) e confirmada por (CAMPOS, 2010), a seção sísmica na Baía de Aratu em que observa-se a liberação de gás apresenta um padrão predominantemente enquadrado no tipo cortina acústica. Há um mascaramento completo abaixo dessa cortina, impossibilitando caracterizações estratigráficas do meio como acamamento sedimentar, embasamento, etc.

Correlacionando a localização das imagens sonográficas com o mapa de distribuição de fácies texturais proposto por LESSA e DIAS (2009), nota-se que a área de trabalho (Figura 1) é caracterizada pelas fácies argila e argila-siltosa. Nas imagens sonográficas (Figura 3) é evidente a presença de uma cobertura sedimentar uniforme e de baixa refletância acústica, correspondentes as tonalidades de cinza claro. Entretanto, há regiões isoladas marcadas por alto contraste acústico (manchas escuras), que podem estar relacionadas ao gás da Baía de Aratu (Figuras 3A, 3B e 3C).

Das feições que evidenciam a presença de gás apresentadas por (JUDD et al., 1997), observa-se plumas de infiltração na coluna d'água nas Figuras $3 \mathrm{~A}$ e $3 \mathrm{~B}$ e feições altamente refletivas na Figura 3C. A correlação da sonografia com os perfis sísmicos confirmou que as manchas nas Figuras 3A e 3B coincidem com as regiões de exsudação de gás, e as feições altamente refletivas na Figura 3C são interpretadas como a resposta acústica do afloramento do embasamento acústico observado no perfil sísmico. A resposta do afloramento rochoso é marcada por um alto contraste acústico com alta rugosidade, podendo ser relacionada ao alto coeficiente de reflexão das rochas.

Em geral regiões onde ocorre exsudação de gás são caracterizadas por alto espalhamento acústico do sinal do sonar de varredura (De BEUKELAER et al (2003); SAGER et al (2004)). 

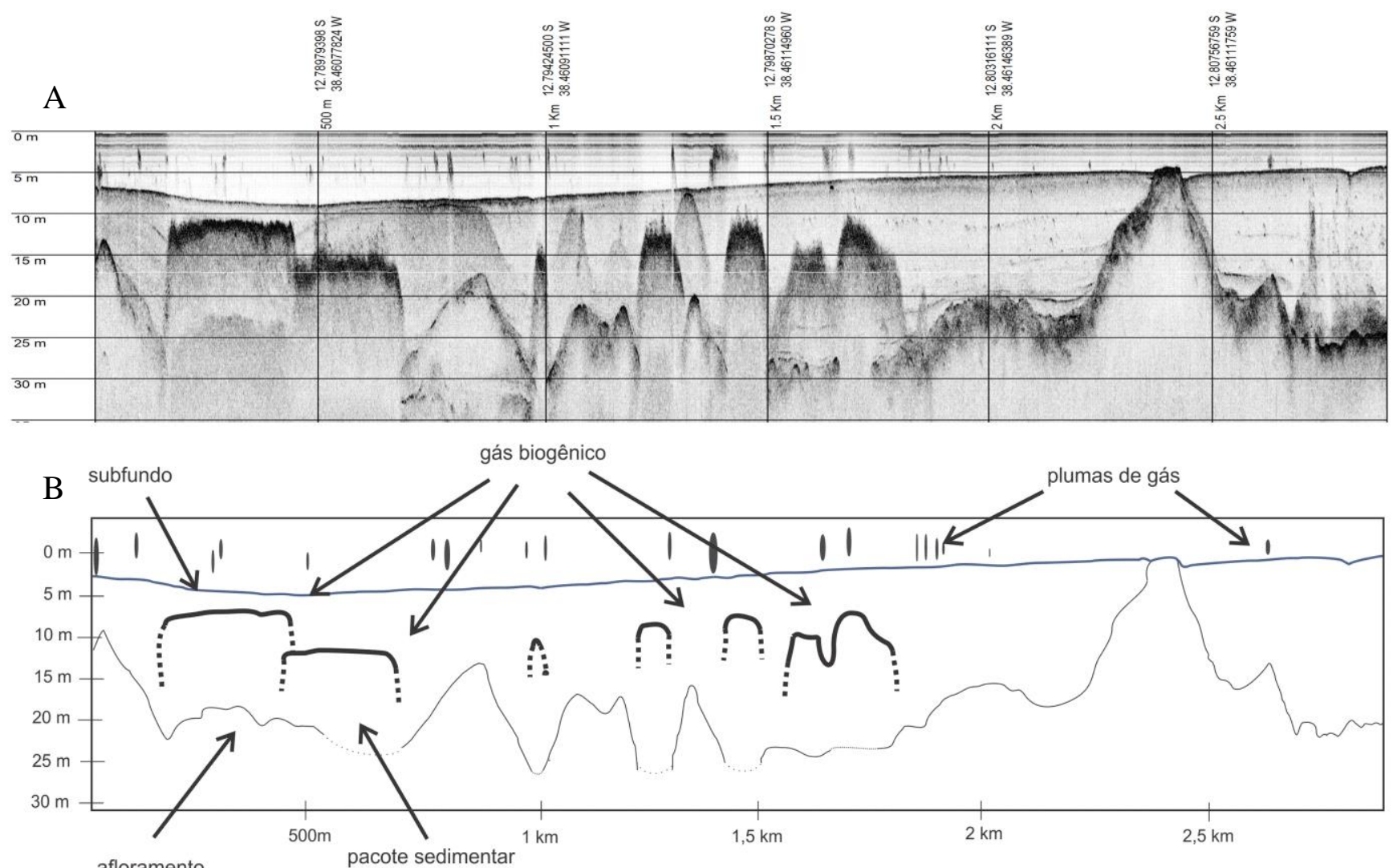

Figura 2 - (A) Perfil obtido a partir do Perfilador de Subfundo. (B) Modelo gerado para auxiliar na interpretação.
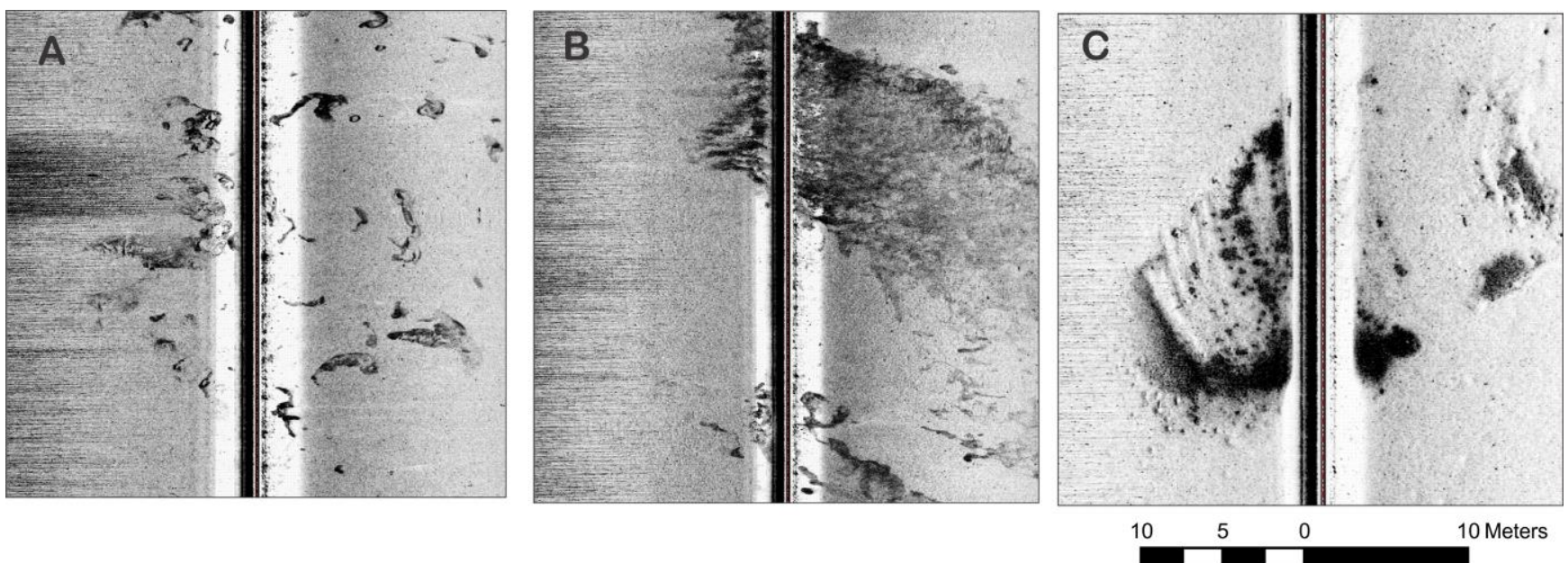

Figura 3 - (A) e (B) Plumas de infiltração na coluna d'água. (C) Resposta acústica do afloramento confirmada pelo Perfilador de Subfundo.

\section{Conclusões}

A aplicação de métodos geofísicos aquáticos confirmou ser eficiente para identificação de regiões marcadas pela exsudação rasa de gases na Baía de Aratu. A interpretação dos registros sísmicos possibilitou a identificação de feições quais: embasamento rochoso, pacote sedimentar, cortinas e plumas associadas às exsudações de gás, com penetração do sinal acústico até $20 \mathrm{~m}$ abaixo do fundo.

As imagens do sonar possibilitaram visualizar a dispersão do gás ao longo da lâmina d'água, bem como representar a distribuição superficial dos sedimentos.

Para uma caracterização precisa da procedência do gás registrado nos dados geofísicos bem como sua 
correlação com os aspectos geológicos, propõe-se a coleta de testemunhos para análise direta e a realização de investigações geofísicas de maior detalhe.

\section{Agradecimentos}

Ao Laboratório de Geofísica Aplicada (LGA/IG/UnB) pela disponibilização dos equipamentos geofísicos. À Phygeo Empresa Junior dos estudantes de graduação em Geofísica da Universidade de Brasília pela colaboração na execução do projeto. E ao Macsimiliano Maly da empresa SALT - Sea \& Limno Technology pela aquisição dos dados do sonar de varredura lateral apresentados nesse trabalho.

\section{Referências}

BITTENCOURT, A. C. S. P., BRICHTA, A.; DI NAPOLI, E. A sedimentação na Baía de Aratu. Revista Brasileira de Geociências, v. 4 n. p. 51-63,1974.

De BEUKELAER, S. M., MACDONALD, I. R., GUINNASSO Jr., N. L., MURRAY, J. A. Distinct side-scan sonar, RADARSAT SAR, and acoustic profiler signatures of gas and oil seeps on the Gulf of Mexico slope. GeoMar Lett, 23: 177-186. (2003)

CAMPOS, R. H. S. Ocorrências De Gás Nos Sedimentos Holocênicos Da Baía De Todos Os Santos: Origem E Distribuição. 2010.

DAVIS, A. M. Shallow gas: an overview. Continental Shelf Research, v. 12, n. 10, p. 1077-1079, 1992.

DIAS, K. A. Estudos das Texturas Sedimentares da Superfície de Fundo Oceânico da Baía de Todos os Santos. Trabalho Final de Graduação, 2003.

GARCIA-GIL, S.; VILAS, F.; GARCIA-GARCIA, A. Shallow gas features in incised-valley fills (Ría de Vigo, NW Spain): a case study. Continental Shelf Research, v. 22, n. 16, p. 2303-2315, nov. 2002.

HATJE, V.; DE ANDRADE, J. B. Baía de Todos os Santos : aspectos oceanográficos. [s.I: s.n.].

JUDD, A. et al. Contributions to atmospheric methane by natural seepages on the UK continental shelf. Marine Geology, v. 137, n. 1-2, p. 165-189, 1997.

JUDD, A. G. Natural seabed gas seeps as sources of atmospheric methane. Environmental Geology, v. 46, n. 8, p. 988-996, 25 nov. 2004.

JUDD, A. G.; HOVLAND, M. The evidence of shallow gas in marine sediments. Continental Shelf Research, v. 12, n. 10, p. 1081-1095, 1992.

LEIFER, I.; MACDONALD, I. R. Dynamics of the gas flux from shallow gas hydrate deposits interaction between oily hydrate bubbles and the oceanic environment. Earth Planet Sci Lett, 210:411-424, 2003.

LESSA, G.; DIAS, K. Distribuição espacial das litofácies de fundo da Baía de Todos os Santos. Quaternary and Environmental Geosciences, v. 01, n. 2, p. 84-97, 2009.

SAGER, W. W.; MACDONALD, I. R.; HOU, R. Side-scan sonar imaging of hydrocarbon seeps on the Louisiana continental slope. AAPG Bulletin, v. 88, n. 6, p. 725-746, jun. 2004. 